\title{
Three-dimensional Ultrasound of the Fetal Brain
}

\author{
Vincenzo D’Addario, Vincenzo Pinto, Luca Di Cagno, Armando Pintucci
}

Dept Obstetrics and Gynecology, University of Bari, Italy

Correspondence: Vincenzo D’Addario, Dept of Obstetrics and Gynecology, Medical School University of Bari

Policlinico, Piazza G. Cesare, 70124 Bari, Italy, e-mail: v.daddario@gynecology4.uniba.it

\begin{abstract}
Three-dimensional ultrasound is the most innovating and attracting modality in the field of ultrasound imaging and represents a superb tool to perform an accurate fetal neuroscan. Once the fetal brain has been scanned, it is then possible to "navigate" in the stored volume choosing among the multiple scanning planes on the three orthogonal spatial axes. Last generation 3D equipments have multiple software facilities which are extremely useful to correctly evaluate the fetal brain such as the multiplanar view, the tomographic ultrasound imaging (TUI), the volume contrast imaging in the C plane (VCI-C plane), the volume calculation, the surface rendering, the $3 \mathrm{D}$ color and power Doppler. Thanks to these imaging modalities it is possible to evaluate the finest anatomical details of the developing brain and to increase the diagnostic accuracy when an abnormal sonographic finding of the fetal brain is recognized during the routine examination.
\end{abstract}

Key words: Three-dimensional ultrasound, fetal brain, brain malformations.

\section{INTRODUCTION}

The development of the fetal brain is a complex mechanism producing remarkable changes of its sonographic appearance throughout pregnancy. Most of the brain structures, however, are already developed at the time when the anomaly scan is performed and a great number of central nervous system (CNS) anomalies may be suspected thanks to a simple screening procedure based on the use of two transabdominal axial scans. The first one passes trough the cavum septi pelluci and the atria of the lateral ventricles (transventricular scan); the second one trough the posterior fossa and the cerebellum (transcerebellar scan). These two simple axial scans offer to the operator a panoramic view of multiple structures of the fetal head (skull, cerebral hemispheres, interhemispheric structures, lateral ventricles, arachnoidal spaces, cerebellum, cisterna magna) and allow him the possibility to suspect the presence of several CNS anomalies.

The recognition of an abnormal sonographic finding during the screening procedure is an indication to a more comprehensive fetal "neurosonography" in order to obtain an accurate diagnosis of the brain anomalies and to properly counsel the parents.

A complete neurosonography relies on multiple parallel scans on the axial, coronal and sagittal planes which offer precious information useful to the final diagnosis. The transabdominal approach to the fetal head has several obstacles to an accurate neuroscan such as the maternal abdominal wall, the placenta, the fetal cranial bones and the head position. A transvaginal approach to the fetal brain was introduced in the early 90 's. ${ }^{1}$ Using the fontanelles and/or the sagittal suture as ultrasound windows serial oblique sections on the coronal and sagittal planes may be obtained revealing the brain morphology in detail. In such a way the operator may mentally reconstruct a three-dimensional view of the fetal brain.

\section{D SONOGRAPHIC ASSESSMENT OF FETAL BRAIN}

\section{Technique}

Three-dimensional ultrasound is the most innovating and attracting modality in the field of ultrasound imaging and represents a superb tool to perform an accurate fetal neuroscan. Thanks to dedicated transabdominal or transvaginal probes a motor driven automatic sweeping is produced in a matter of seconds with the acquisition of a region of interest (ROI) in the target organ. It is then possible to "navigate" in the stored volume choosing among the multiple scanning planes on the three orthogonal spatial axes. ${ }^{2,3}$ Last generation $3 \mathrm{D}$ equipments have multiple software facilities which are extremely useful to correctly evaluate the fetal brain:

- Multiplanar view: It allows the simultaneous visualization of the three spatial planes (axial, sagittal and coronal) intersecting in a dot of the stored volume. The operator can move the intersecting dot in each of the three planes thus obtaining an infinite series of images useful to a complete evaluation of the brain. When the dot is located in the cavum septi pellucidi on the axial plane, the structures close to it, such as the corpus callosum and the frontal horns are simultaneously displaced on the other two planes (Fig. 1).

- Tomographic ultrasound imaging (TUI): It allows the simultaneous visualization of multiple parallel slices in one of the three orthogonal planes in a way similar to CT or MRI (Fig. 2).

- Volume contrast imaging (VCI): It allows obtaining a thick slice of the fetal brain with the simultaneous real time visualization of two orthogonal planes. This is particularly 


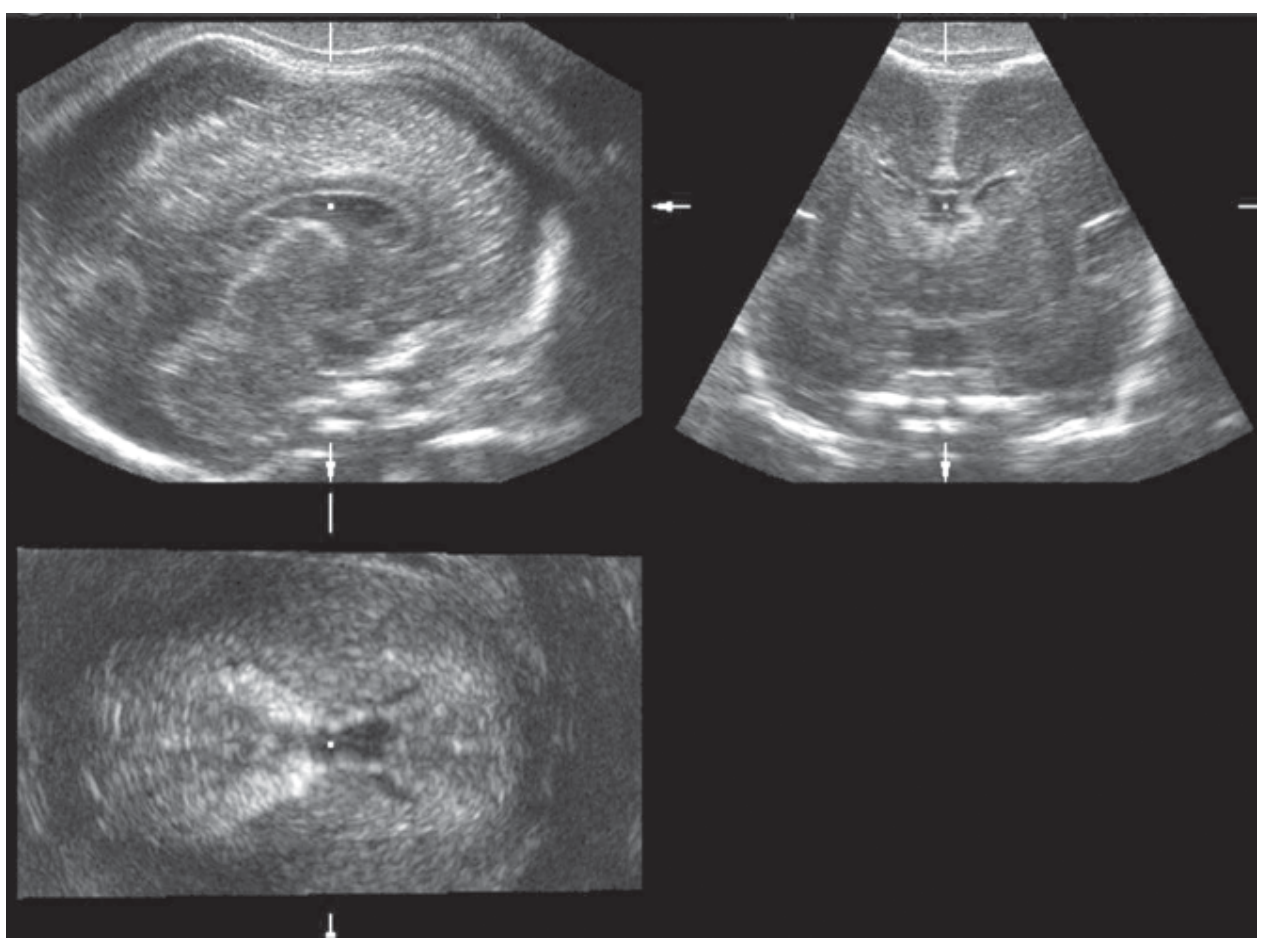

Fig. 1: 3D multiplanar transvaginal view of a normal brain at 22 weeks of gestation. Sagittal (left upper), coronal (right upper) and axial (left lower) images are simultaneously displaced. The intersecting dot is located in the cavum septi pellucidi. The corpus callosum and the cerebellar vermis can be recognized in the sagittal view. The frontal horns of the lateral ventricles clearly visible in the coronal view. The choroids plexuses may be recognized in the axial view. The cavum septi pellucidi, where the intersecting dot is located, is visible in all three views

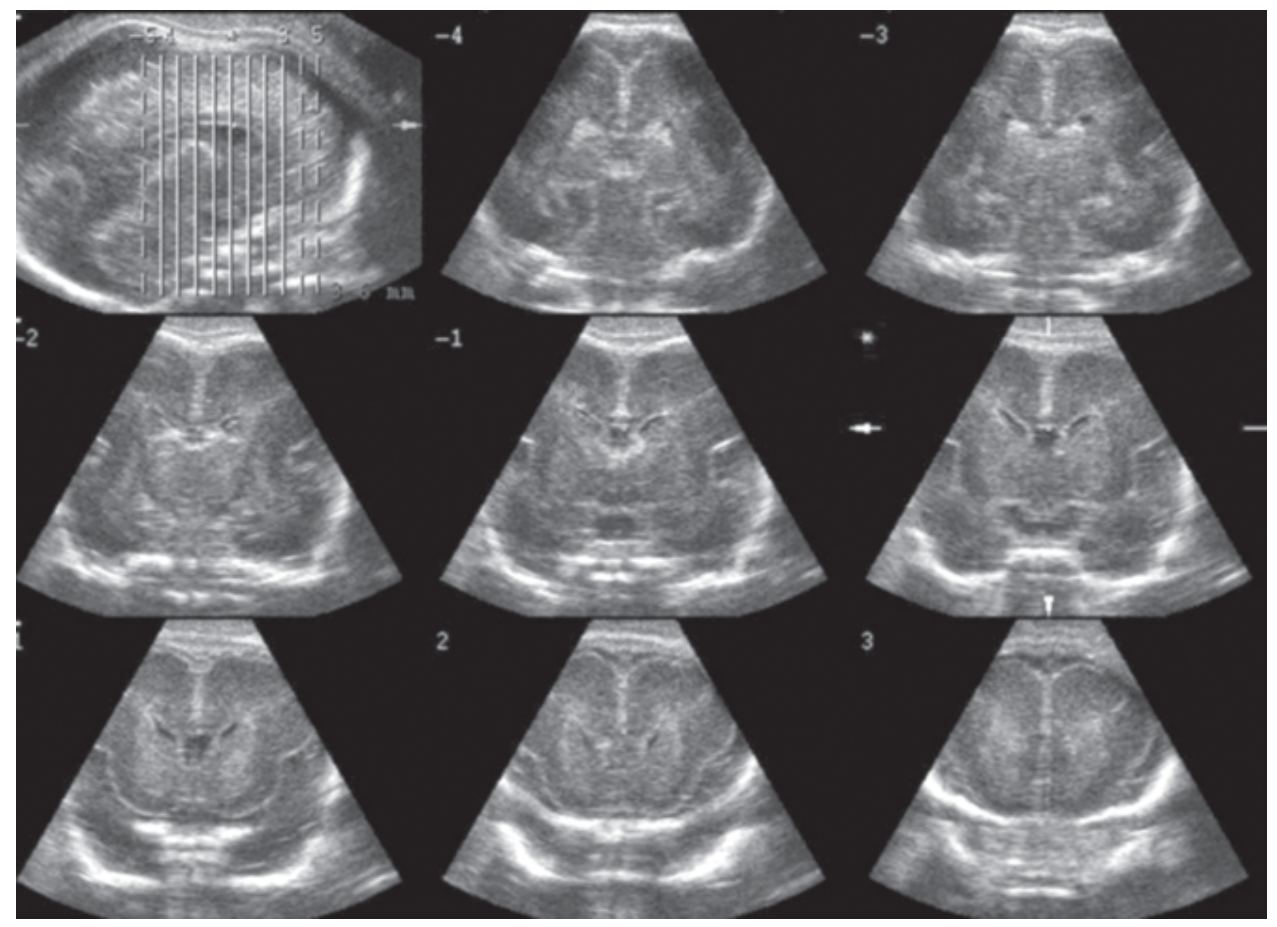

Fig. 2: Tomographic ultrasound imaging (TUI) of a normal brain at 22 weeks of gestation. Multiple parallel scans on the coronal plane are simultaneously displaced starting from the posterior (top row, middle) to the anterior aspect of the corpus callosum (low row, right). Fine anatomical details, such as the foramen of Monro (middle row, middle) and wide insula (middle row, right) are clearly demonstrated 


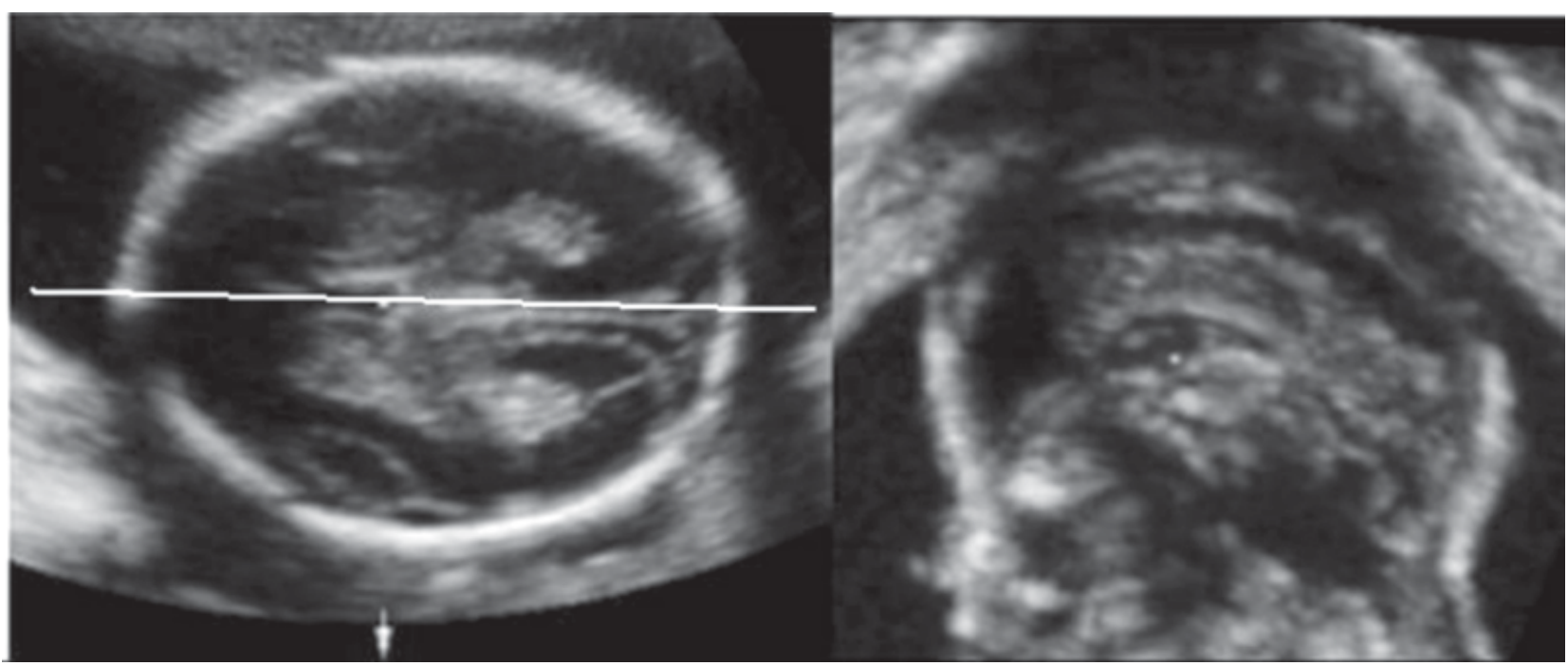

Fig. 3: Volume contrast imaging ( $\mathrm{VCl})$ in the C-plane: In the axial scan a line is selected passing trough the cavum septi pellucidi between the two hemispheres. The sagittal view (C-plane) orthogonal the axial one is simultaneously displaced showing the corpus callosum

useful to image the corpus callosum on the reconstructed sagittal plane once the cavum septi pellucidi is visualized on the axial view (Fig. 3).

- Volume calculation: It allows the extraction and volumetric evaluation of targets structures such as hemispheres, ventricles, cerebellum, and intracranial lesions (Fig. 4).

- Surface rendering: It allows the surface visualization of structures such as the calvarium and the sutures (Fig. 5).

\section{Normal Brain Development}

Using transvaginal 3D probes it is possible to evaluate the fetal brain starting from its early stages of development. The first report on the 3D reconstruction of the early brain vesicles was by Blaas and coll. ${ }^{4}$ in 1995; using a system based on a transvaginal transducer attached to a PC-based TomTec EchoScan Unit they obtained high-resolution three-dimensional images of the brain cavities. The last generation 3D equipments offer this possibility in a matter of second, thus allowing the diagnosis of some brain anomalies in the first trimester. ${ }^{5}$

As the pregnancy progresses the brain undergoes complex modifications continuing throughout gestation till after birth. The main developmental milestones of the fetal brain during the second trimester are:

1. The variation in volume and size of the lateral ventricles with the differentiation of the frontal, occipital and temporal horns.

2. The development of the corpus callosum.

3. The development of the cerebellar vermis. With the different imaging modalities of 3D sonography it is possible to evaluate in details these developmental events.

Timor-Tritsch and coll ${ }^{6}$ described the so-called "three horns view" of the lateral ventricles, depicting the anterior, posterior,

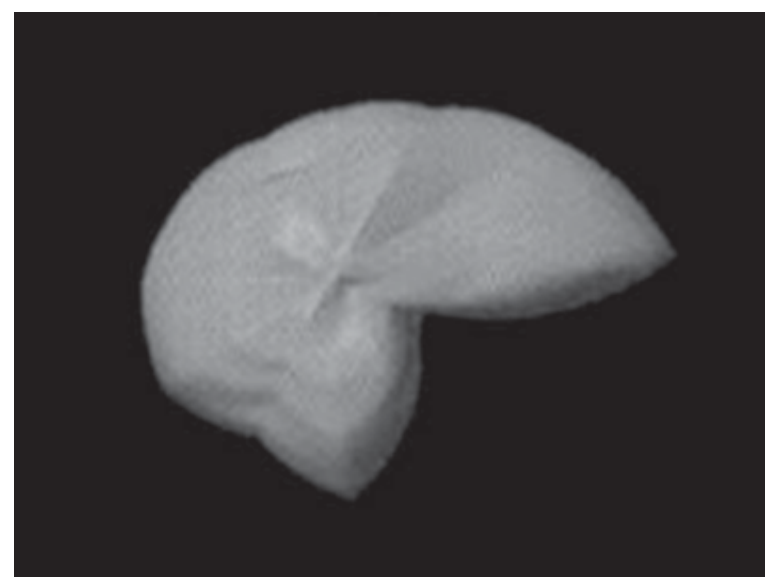

Fig. 4: 3D volume extraction and volumetric analysis of a dilated lateral ventricle

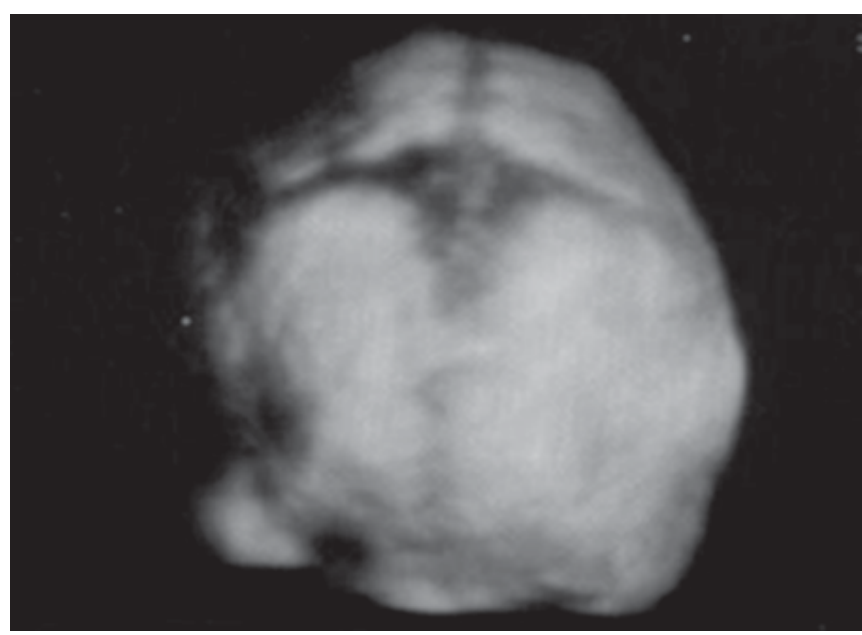

Fig. 5: Surface rendering of the metopic suture 
and inferior horns on the same image, which is easy to obtain using both the $2 \mathrm{D}$ and the $3 \mathrm{D}$ ultrasound techniques. It provides diagnostic and clinically useful information much like neonatal transfontanelle ultrasound imaging after which it is emulated (Fig. 6). Pilu and coll ${ }^{7}$ compared median planes obtained by 2D sonography with those reconstructed using 3D modality from volumes acquired from transabdominal axial planes of the fetal head (3D median planes), by either multiplanar analysis of static volumes or volume contrast imaging in the coronal plane (VCIC) and found a good correlation between 2D and 3D images (Fig. 7). Vianls and coll ${ }^{8}$ used the VCI-C plane mode to evaluate the normal appearance of the fetal cerebellar vermis and developed nomograms of the vermian height, width and surface. By measuring the three cerebellar diameters the cerebellar volume may be calculated. ${ }^{9}$ Further measurements were proposed by Paladini and Volpe ${ }^{10}$ who suggested to measure the tentorovermian angle, tentoroclivus angle, clivovermian angle ad vermian diameter/biparietal diameter $\times 100$ ratio (VD/ $\mathrm{BPD}$ ratio) to differentiate different pathologies affecting the cerebellar vermis.

During the third trimester the brain development is mainly characterized by the mechanism of neuronal proliferation, migration and differentiation which bring to the development of gyri and sulci and to the brain volume increase. Brain volume can be easily measured by $3 \mathrm{D} .{ }^{11}$ Roelfsema and coll ${ }^{12}$ measured a nearly 10-fold increase of the fetal brain volume during the second half of gestation with acceptable intraobserver variability.

Sonographic examination of the brain surface with the aid of the multiplanar view and TUI may help to accurately demonstrate structures of the fetal cortex. Accurate knowledge of the ultrasound appearance of the fetal cortex at different stages of gestation is important in order to be able to diagnose in-utero malformations of cortical development. ${ }^{13,14}$

Brain vascularization may be evaluated by the use of Color or Power Doppler modality. The combination 3D and sonoangiography shows the brain circulation three-dimensionally. ${ }^{15}$

Finally, using the rendering mode and the transparent maximum mode a clear view of the cranial bones and suture may be achieved ${ }^{16}$ (Fig. 5). This technique has proved to be useful also in the evaluation of the skull base development. Serial 3D sonographic measurements of anterior skull base length, posterior cranial fossa length and skull base angle were made in normal singleton pregnancies at 18-34 weeks of gestation by Roelfsema and coll ${ }^{17}$ A statistically significant gestational agerelated increase was established for both the anterior skull base length and the posterior cranial fossa length and the skull base angle showed a small but significant flexion of about 6 degrees. A higher increment in posterior cranial fossa length relative to anterior skull base angle was established as a consequence of the brain growth. The same author suggested a 3D technique to evaluate the craniofacial pattern profile and introduced a

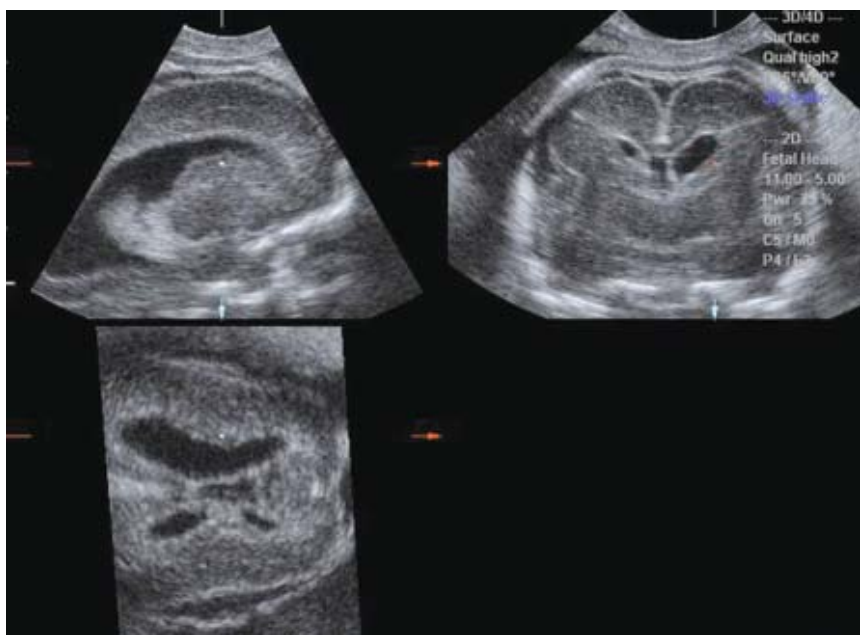

Fig. 6: Multiplanar view in a case of unilateral ventriculomegaly. The sagittal scan (left upper) shows the three horns view of the lateral ventricle, depicting the anterior, posterior, and inferior horns on the same image. The choroid plexus is seen in the region of the atrium and inferior horn

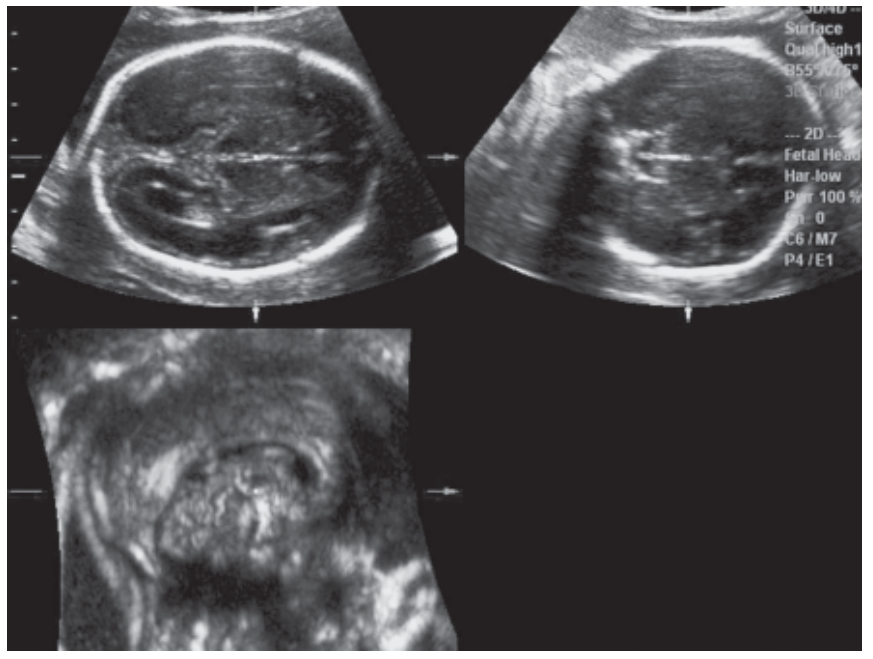

Fig. 7: 3D reconstruction of the midsagittal plane showing the corpus callosum and the cerebellar vermis

craniofacial variability index (CVI) which can assist in the evaluation of fetal facial anatomy for distinguishing between normal and abnormal craniofacial development. ${ }^{18}$

The advantages of 3D ultrasound in the evaluation of the fetal brain are undoubted and the routinary use of transabdominal 3D acquisition of an ultrasound volume of the fetal head with later analysis of the entire brain anatomy using the multiplanar images has been suggested by Correa and coll. ${ }^{19}$ They were able to obtain acceptable cerebral multiplanar images in $92 \%$ of the cases. The corpus callosum could be seen in $84 \%$ of the patients, the fourth ventricle in $78 \%$, the lateral sulcus (Sylvian fissure) in $86 \%$, the cingulate sulcus in $75 \%$, the 
cerebellar hemispheres in $98 \%$, the cerebellar vermis in $92 \%$ and the medulla oblongata in $97 \%$; the thalami and the cerebellopontine cistern (cisterna magna) were identified in all cases; at or beyond 20 weeks, superior visualization (in $>90 \%$ of cases) was achieved of the cerebral fissures, the corpus callosum (97\%), the supracerebellar cisterns $(92 \%)$ and the third ventricle $(93 \%)$.

\section{Brain Malformations}

Several papers have been reported on the use of 3D sonography in the diagnosis of fetal brain anomalies. Although the diagnosis of each CNS anomaly was made using conventional twodimensional sonography, 3D sonography proved most helpful delineating the exact nature and anatomic level of the anomaly.

3D sonography is particularly useful in the evaluation of the midline anomalies, thanks to the reconstruction of the midsagittal plane by using the multiplanar or VCI mode. The midsagittal view of the fetal brain offers the simultaneous visualization of structures such as the corpus callosum and the cerebellar vermis. We have demonstrated that the combination of sonographic appearance of these two structures is helpful in recognizing the cause of ventriculomegaly. ${ }^{20}$ Failure to demonstrate the corpus callosum is typical of corpus callosum agenesis (complete or partial) (Fig. 8); a small and funneling posterior fossa is typical of Chiari II malformation (Fig. 9); an hypoplastic and upward rotated cerebellar vermis with enlarged fourth ventricle is a sign of Dandy-Waker malformation (Fig. 10); the combination of normal corpus callosum and normal cerebellar vermis is an sign of isolated ventriculomegaly, which is mainly due to aqueduct stenosis (Fig. 11). Although the quality of the reconstructed midsagittal plane is lower than the direct 2D visualization of the same plane, however 3D images are usually obtained more easily and quickly particularly when the position of fetal head does not allow a direct 2D visualization.

Fetal cerebral ventriculomegaly may also be evaluated using the TUI mode which offers multiple parallel section at different level in the axial, coronal and sagittal planes (Fig. 12) producing images which are similar to the CT and MRI. These are useful when consulting the pediatric neuroradiologist and neurosurgeon who are more familiar with these imaging techniques.

A further useful application of 3D sonography is the evaluation of the posterior fossa and particularly of the cerebellar vermis, fourth ventricle and cerebellopontine cistern. Upward rotation of the vermis, upward displacement of the tentorium and underdevelopment of the vermis are the main features of posterior fossa abnormalities and can accurately evaluated in midsagittal view of the posterior fossa (Fig. 13). By measuring the tentorovermian angle, the tentoroclivus angle, the clivovermian angle and vermian diameter/biparietal diameter $\times$ 100 ratio (VD/BPD ratio), Paladini and Volpe accurately

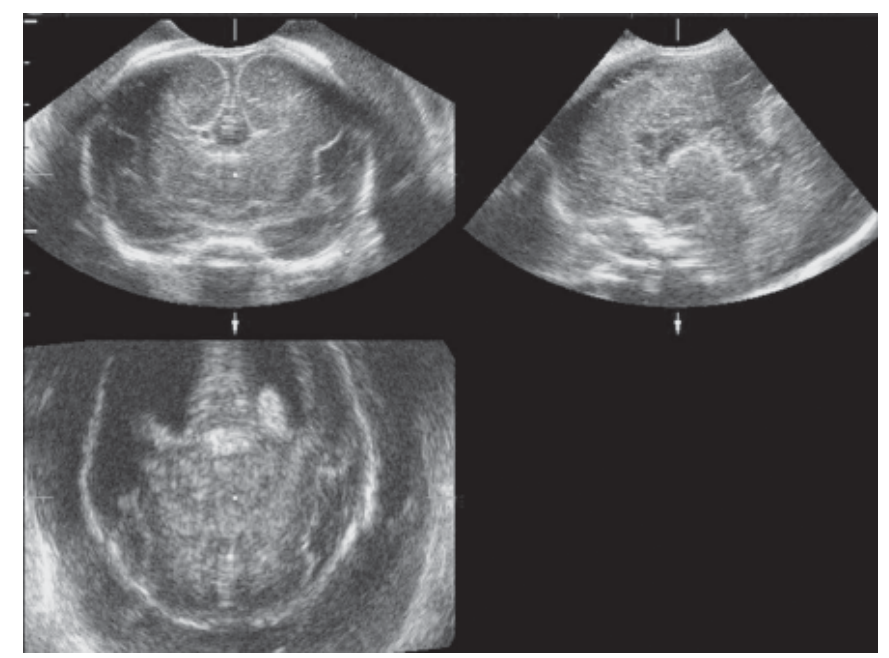

Fig. 8: Multiplanar view in a case of corpus callosum agenesis. In the coronal view (left upper) the typical "bull's head" appearance of the frontal horns is visible. The sagittal view (right upper) shows the absence of the corpus callosum. A mild dilatation of the lateral ventricles is seen in the axial view (left lower)

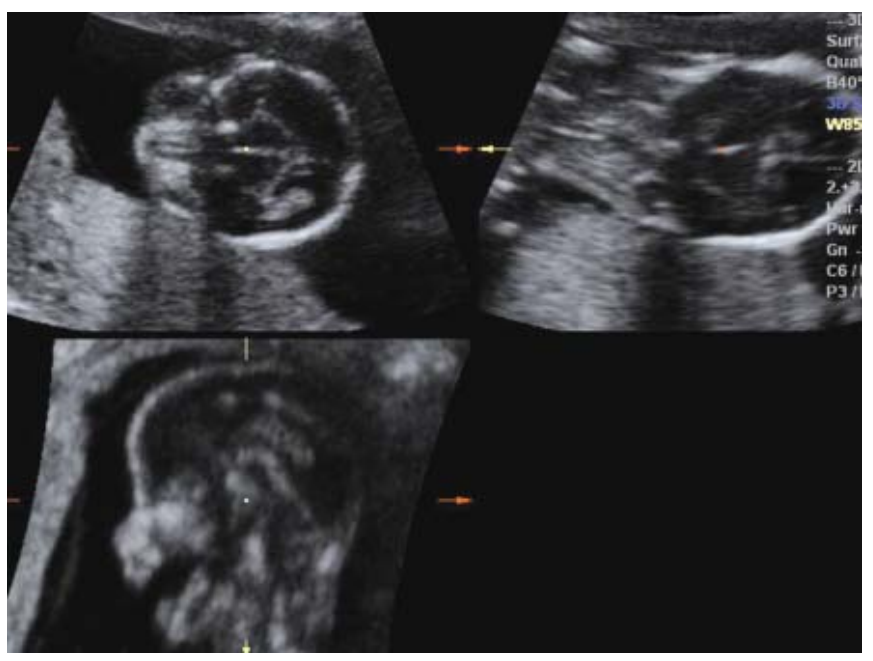

Fig. 9: Multiplanar view in a case of Chiari II malformation. The reconstruction of the sagittal view (left lower) shows the typical funnelling appearance of the posterior fossa

diagnosed 20 cases of posterior fossa anomalies (5 DandyWalker malformations, 8 Dandy-Walker variant and 7 megacisterna magna). ${ }^{10}$ The main limitation of this technique is represented by shadowing produced by the petrous bones during the volume acquisition.

3D sonography may be applied to every CNS abnormality diagnosed with the traditional 2D technique and may offer further information useful to the correct diagnosis. Figures 14 to 18 show some examples of this application. Figure 14 shows a unilateral ventriculomegaly in the multiplanar and surface 


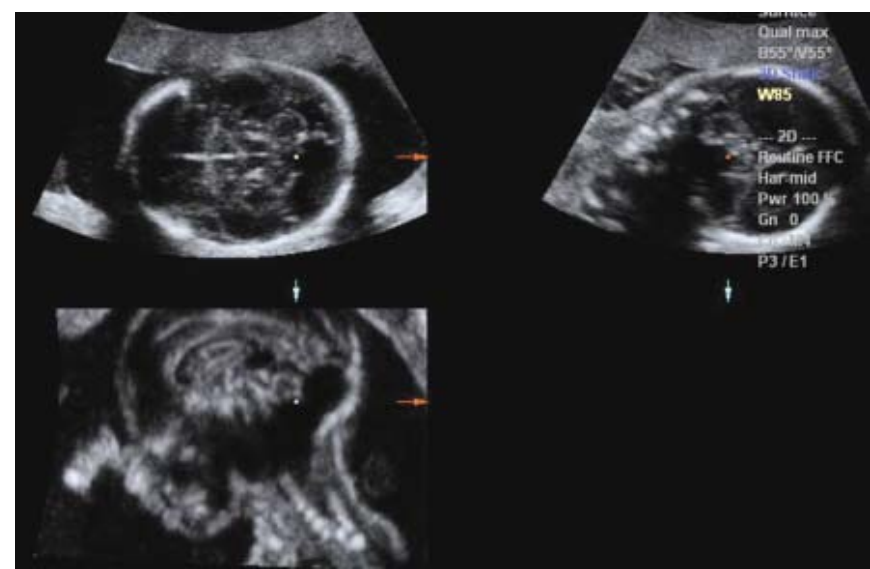

Fig. 10: Multiplanar view in a case of Dandy-Walker malformation. A small vermian defect and a cystic structure behind the cerebellum are seen in the axial view (left upper). The reconstructed sagittal view (left lower) shows the hypoplastic and upper rotated cerebellar vermis with cystic posterior dilatation of the fourth ventricle; the corpus callosum is normal

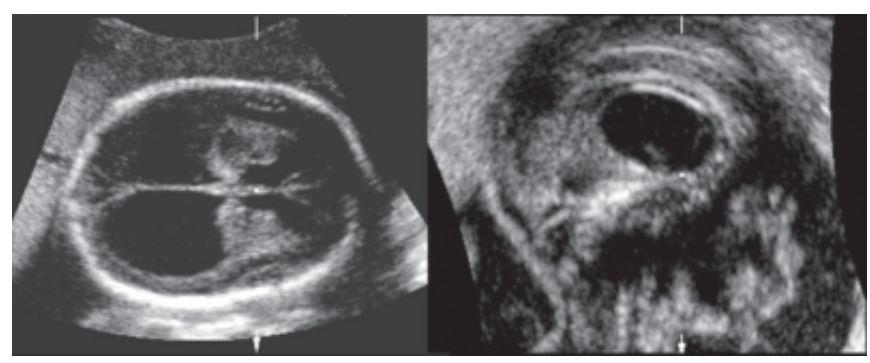

Fig. 11: VCl-C plane in a case of severe isolated ventriculomegaly. The sagittal view shows a wide fenestration of the septum pellucidum with upward displacement of the corpus callosum

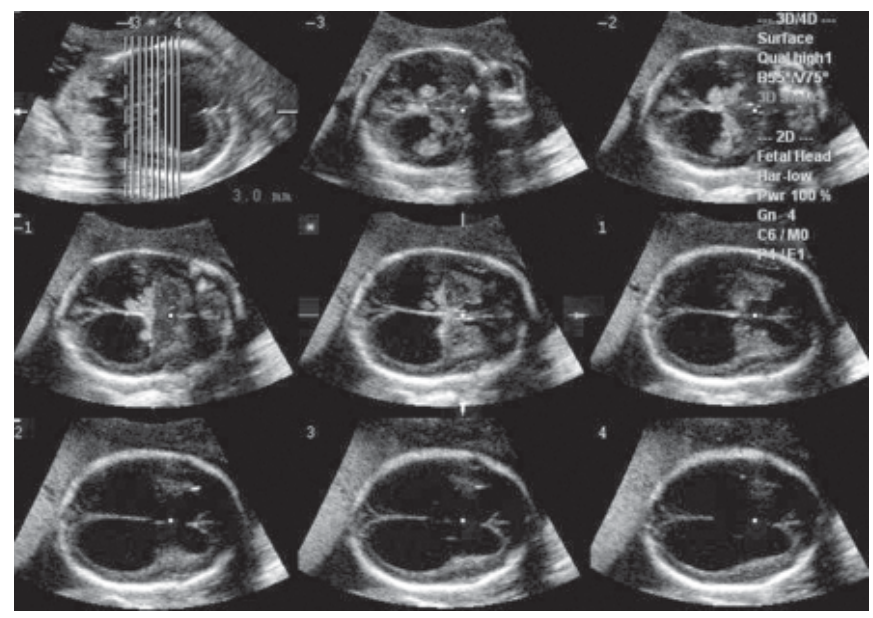

Fig. 12: TUI in the same case of Figure 11. The multiple parallel scans on the axial plane starting from the base of the skull (top row, middle) show the wide communication between the lateral ventricles (low row, right)

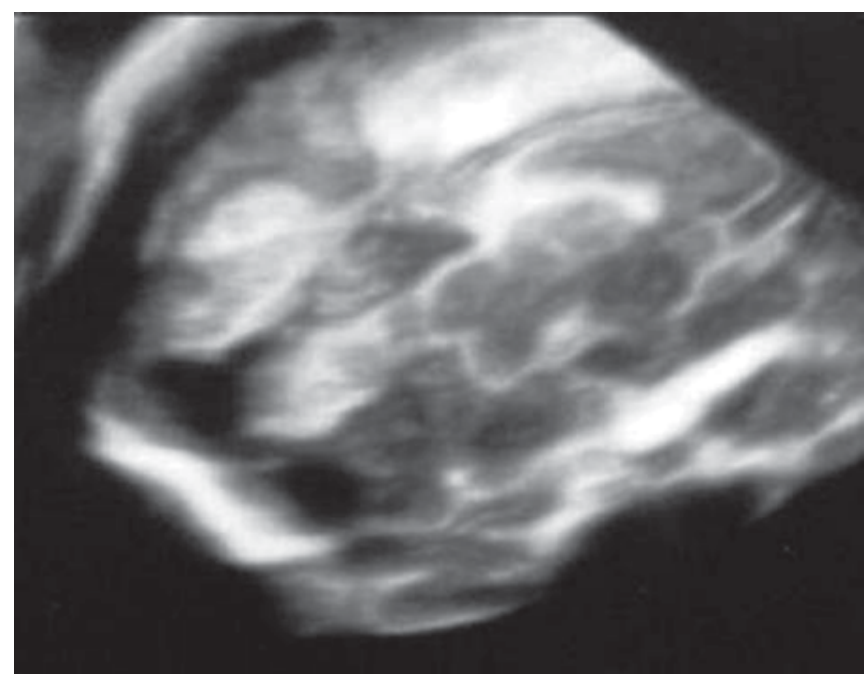

Fig. 13: Surface rendering of the posterior fossa in a fetus with DandyWalker variant. The cerebellar vermis is hypoplastic but is not rotated

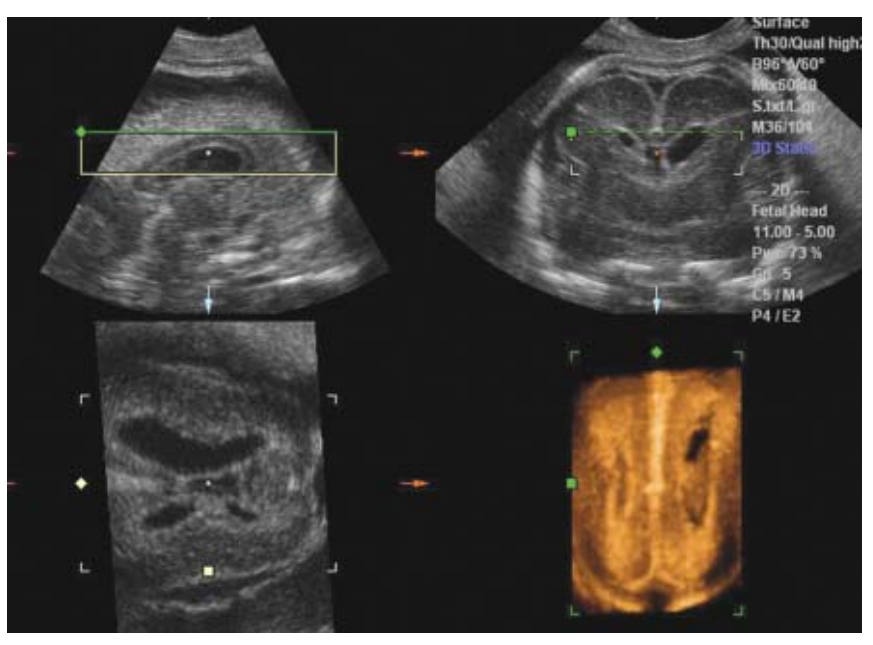

Fig. 14: Mutiplanar and surface rendering mode in a fetus with unilateral ventriculomegaly. The midsagittal view (upper left) shows the normal corpus callosum. The asymmetry of the lateral ventricles may be seen in the coronal view (upper right), in the axial view (lower left) and in the surface rendering mode (lower right)

modalities, confirming the normal development of the corpus callosum. In Figure 15 a wide schizecephaly is represented with the TUI modality to demonstrate the severity of the defect. In Figure 16 a small occipital meningocele is represented with the VCI-C plane mode showing the small bony defect from which only meninges are protruding. Figure 17 shows a huge myelomeningocele in the surface rendering mode. In Figure 18 vein of Galen aneurysm is represented in the 3D color Doppler mode.

A final application of 3D in fetal neurosonology is the possibility to evaluate fetal motorial behavior by the direct 


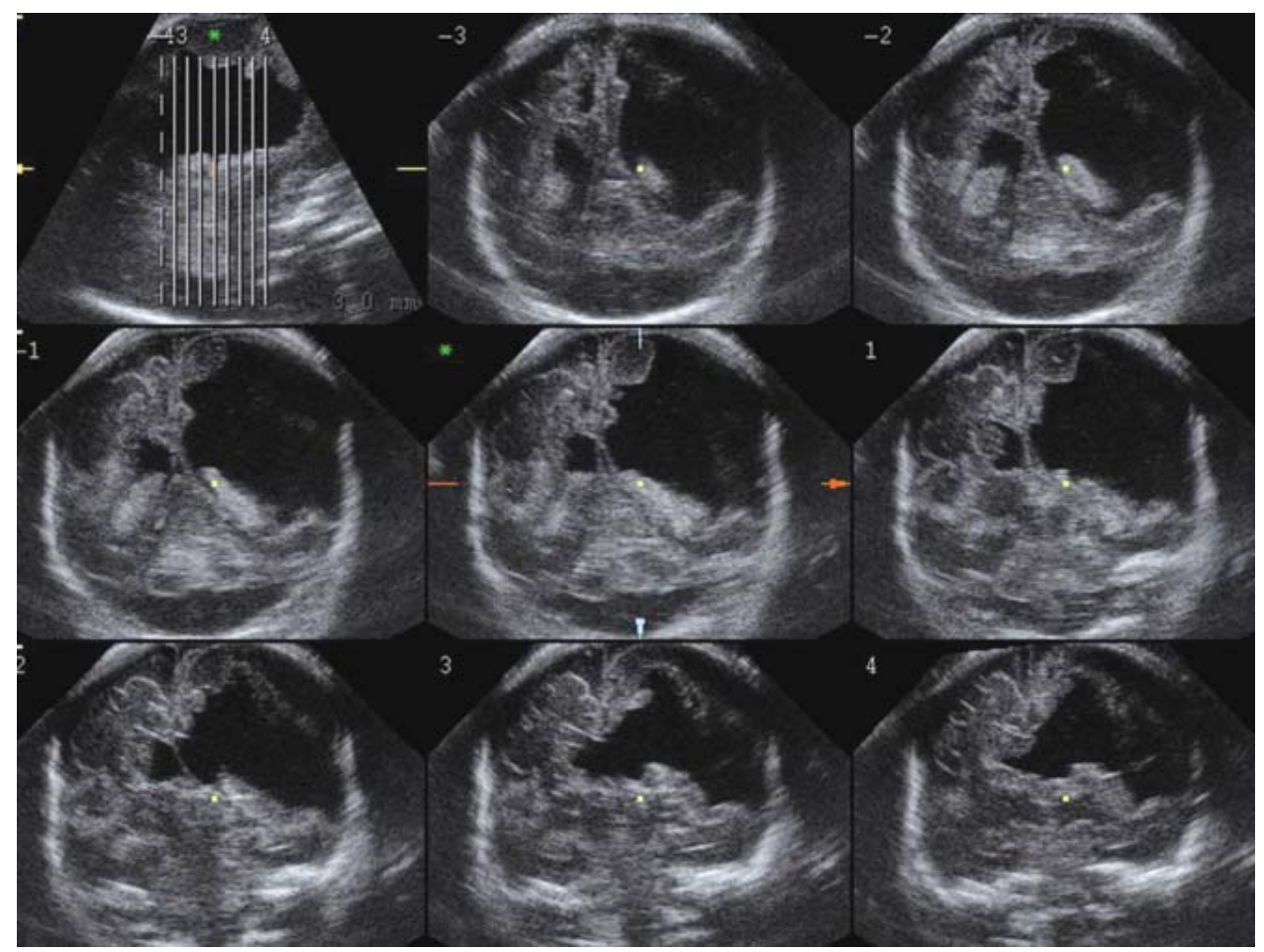

Fig. 15: TUI imaging in the coronal plane in a case of severe schizencephaly. The multiple parallel scans starting from the posterior (middle, top row) to the anterior (right, low row) aspect of the brain show the wide defect of the left hemisphere

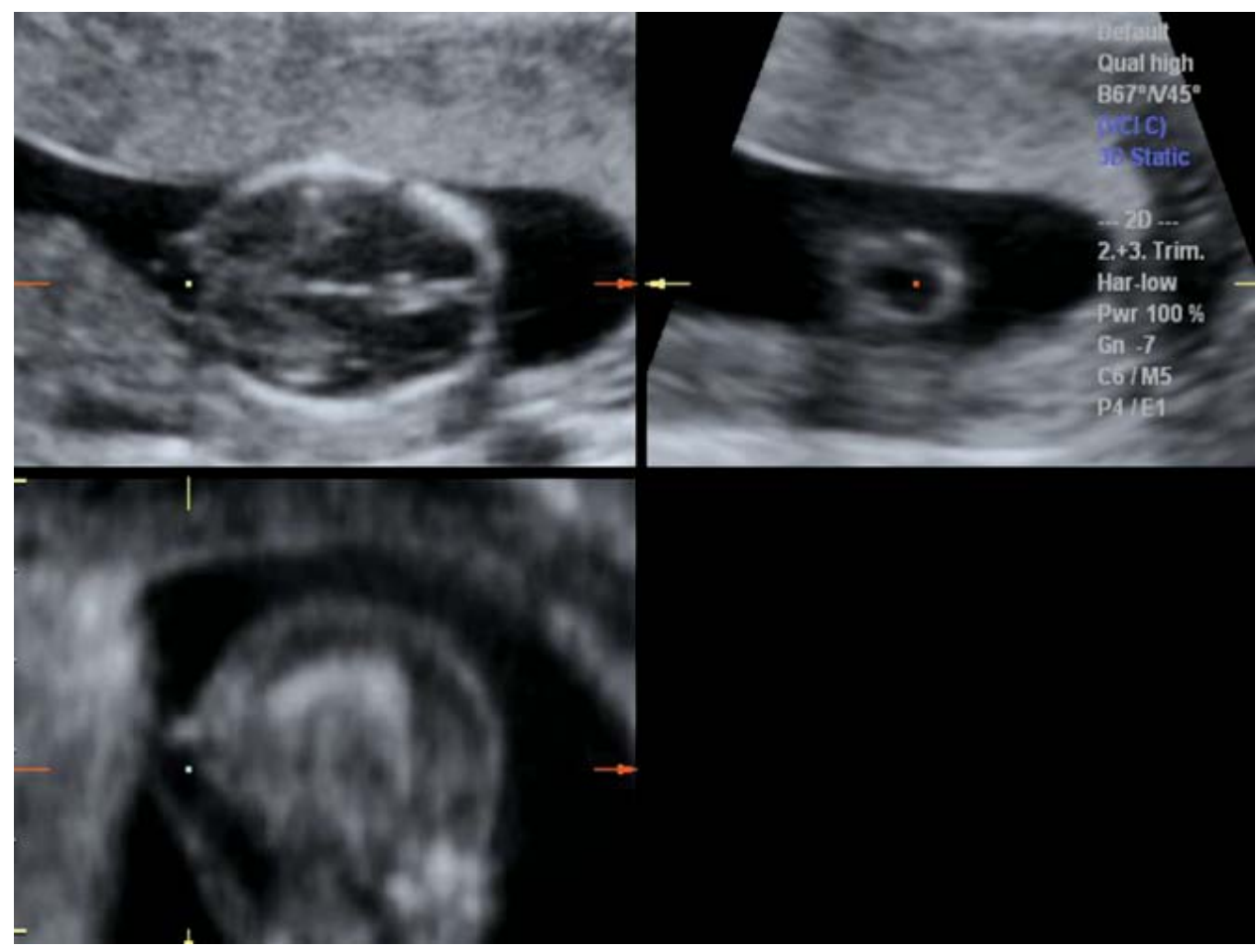

Fig. 16: Multiplanar view in a 14 weeks fetus with a small occipital meningocele 


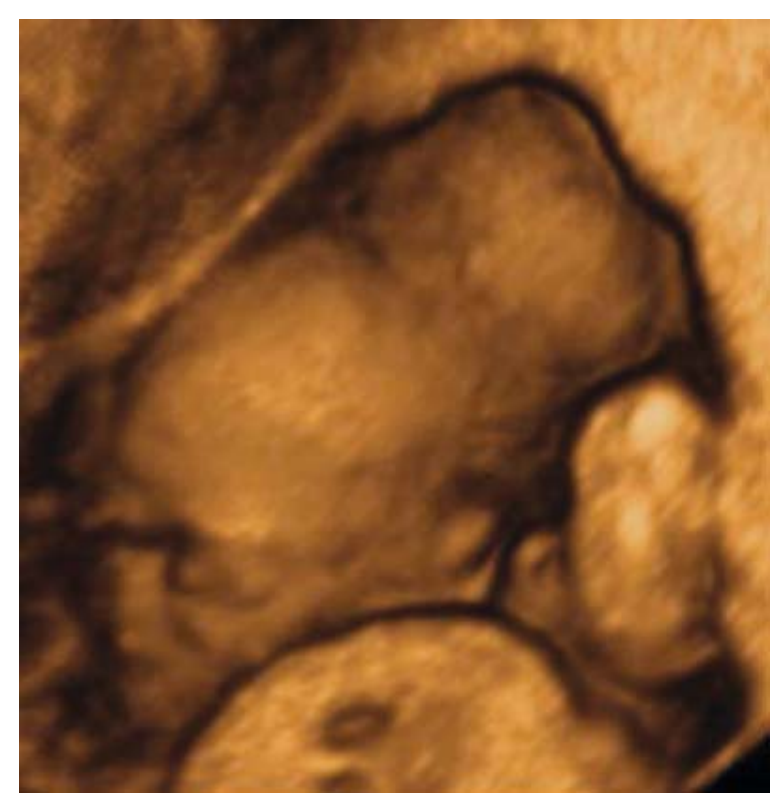

Fig. 17: Surface rendering of a huge myelomeningocele

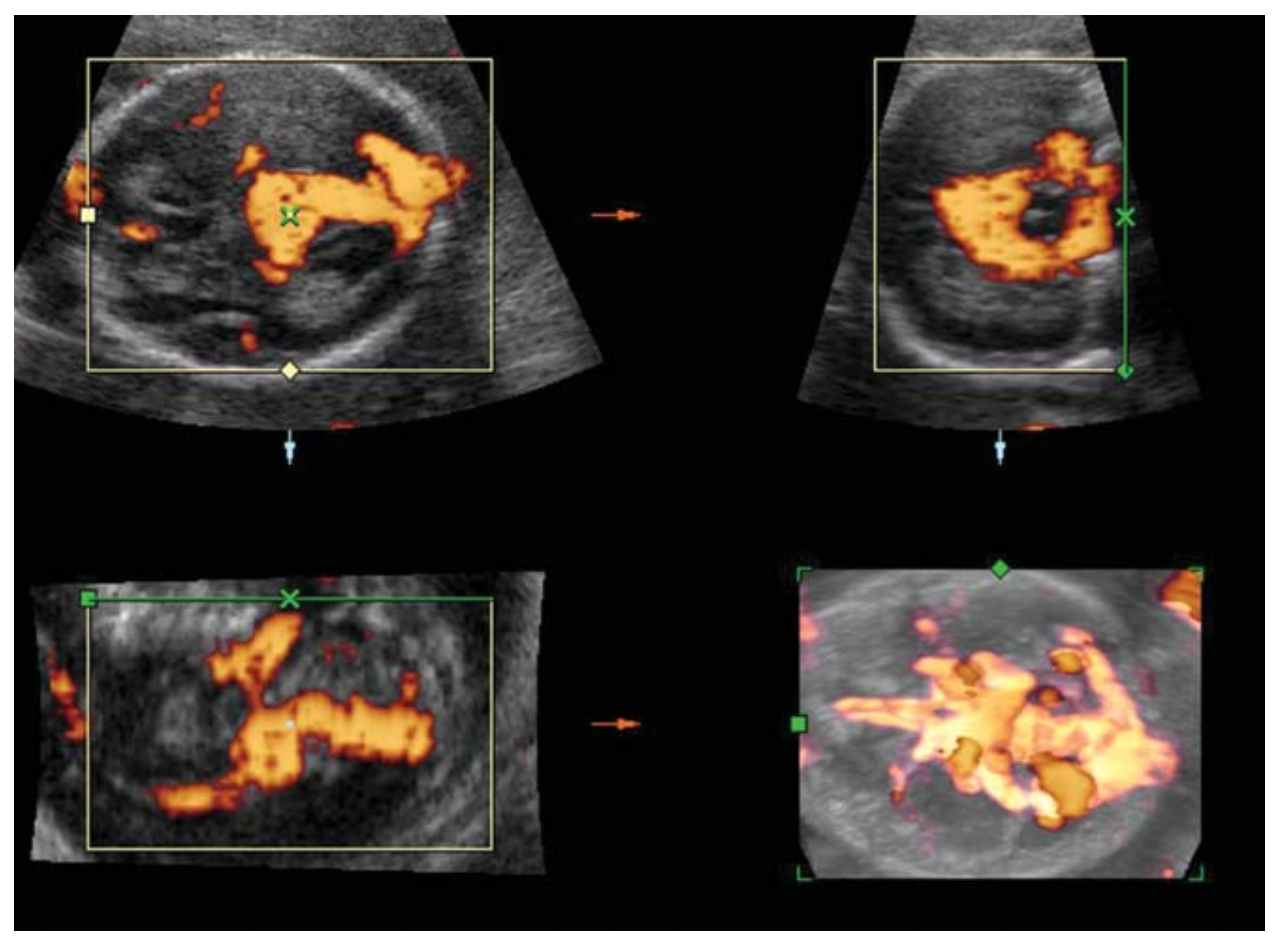

Fig. 18: 3D multiplanar view and power-Doppler imaging of a vein of Galen aneurysm

visualization of fetal movement in the 4D modality. It has been suggested that the assessment of fetal behavioral patterns could give us insight into the integrity of fetal central nervous system and enable the early detection of cerebral dysfunctions. $4 \mathrm{D}$ represents a significant improvement in the assessment of fetal behavior and can open a new perspective for the investigations of fetal behavioral patterns and contribute significantly to our better understanding of complex neurodevelopmental events. Kurjak, et al the pioneers of this new exiting, are of interest and have described patterns of 
neurodevelopmental behavior during the three trimesters of pregnancy using this imaging modality. ${ }^{21,22}$ Abnormal behavioral patterns may be the sign of brain damage and their identification during the second half of pregnancy may serve as a retrospective marker of a prenatal insult. ${ }^{23,24}$

In conclusion 3D sonography is a fundamental tool for a complete neurosonographic examination of the fetus and can help to a better understanding of the complex mechanisms leading to the normal or abnormal brain development.

It must also be considered the great advantage of storing into the computer the volume acquired during the examination and to re-evaluate it in another setting together with other specialists, such as pediatric neuroradiologist and surgeon. Last, but not least, is the possibility to use the stored volumes as a font of teaching.

Even though scientific evidences do not still exist to confirm the efficacy of 3D sonography in improving the clinical outcome in fetuses with brain anomalies, there is no doubt that this technique is extremely useful for specialists involved in fetal neurosonology.

\section{REFERENCES}

1. Monteagudo A, Reuss ML, Timor-Tritsh IE. Imaging the fetal brain in the second and third trimesters using transvaginal sonography. Obstet Gynecol 1991;77:27-32.

2. Monteagudo A, Timor-Tritsch IE, Mayberry P. Threedimensional transvaginal neurosonography of the fetal brain: 'navigating' in the volume scan. Ultrasound Obstet Gynecol 2000;16:307-13.

3. Pooh RK, Pooh KH. Fetal neuroimaging with new technology. Ultrasound Review Obstet Gynecol 2002;2:178-81.

4. Blaas HG, Eik-Nes SH, Kiserud T, Berg S, Angelsen B, Olstad B. Three-dimensional imaging of the brain cavities in human embryos. Ultrasound Obstet Gynecol 1995;5:228-32.

5. Blaas HG, Eik-Nes SH, Vainio T, Isaksen CV. Alobar holoprosencephaly at 9 weeks gestational age visualized by twoand three-dimensional ultrasound. Ultrasound Obstet Gynecol 2000;15:62-5.

6. Timor-Tritsch IE, Monteagudo A, Mayberry P. Threedimensional ultrasound evaluation of the fetal brain: the three horn view. Ultrasound Obstet Gynecol 2000;16:302-6.

7. Pilu G, Segata M, Ghi T, Carletti A, Perolo A, Santini D, Bonasoni P, Tani G, Rizzo N. Diagnosis of midline anomalies of the fetal brain with the three-dimensional median view. Ultrasound Obstet Gynecol 2006;27:522-9.

8. Vinals F, Munoz M, Naveas R, Shalper J, Giuliano A. The fetal cerebellar vermis: anatomy and biometric assessment using volume contrast imaging in the C-plane (VCI-C). Ultrasound Obstet Gynecol 2005;26:622-7.

9. Chang CH, Chang FM, Yu CH, Ko HC, Chen HY. Assessment of fetal cerebellar volume using three-dimensional ultrasound. Ultrasound Med Biol 2000;26:981-8.
10. Paladini D, Volpe P. Posterior fossa and vermian morphometry in the characterization of fetal cerebellar abnormalities: a prospective three-dimensional ultrasound study. Ultrasound Obstet Gynecol. 2006;27:482-9.

11. Chang $\mathrm{CH}$, Yu CH, Chang FM, Ko HC, Chen HY. The assessment of normal fetal brain volume by 3-D ultrasound. Ultrasound Med Biol 2003;29:1267-72.

12. Roelfsema NM, Hop WC, Boito SM, Wladimiroff JW. Threedimensional sonographic measurement of normal fetal brain volume during the second half of pregnancy. Am J Obstet Gynecol 2004;190:275-80.

13. Toi A, Lister WS, Fong KW. How early are fetal cerebral sulci visible at prenatal ultrasound and what is the normal pattern of early fetal sulcal development? Ultrasound Obstet Gynecol 2004;24:706-15.

14. Cohen-Sacher B, Lerman-Sagie T, Lev D, Malinger G. Sonographic developmental milestones of the fetal cerebral cortex: a longitudinal study. Ultrasound Obstet Gynecol 2006;27:494-502.

15. Pooh RK, Pooh K. Transvaginal 3D and Doppler ultrasonography of the fetal brain. Semin Perinatol 2001;25:3843.

16. Faro C, Benoit B, Wegrzyn P, Chaoui R, Nicolaides KH. Threedimensional sonographic description of the fetal frontal bones and metopic suture. Ultrasound Obstet Gynecol 2005;26:61821.

17. Roelfsema NM, Grijseels EW, Hop WC, Wladimiroff JW. Threedimensional sonography of prenatal skull base development. Ultrasound Obstet Gynecol. 2007;29:372-7.

18. Roelfsema NM, Hop WC, van Adrichem LN, Wladimiroff JW. Craniofacial variability index in utero: a three-dimensional ultrasound study. Ultrasound Obstet Gynecol 2007;29:258-64

19. Correa FF, Lara C, Bellver J, Remohi J, Pellicer A, Serra V. Examination of the fetal brain by transabdominal threedimensional ultrasound: potential for routine neurosonographic studies. Ultrasound Obstet Gynecol 2006;27:503-8.

20. D'Addario V, Pinto V, Di Cagno L, Pintucci A. The midsagittal view of the fetal brain: a useful landmark in recognizing the cause of fetal cerebral ventriculomegaly. J Perinat Med 2005;33:4237.

21. Kurjak A, Pooh RK, Merce LT, Carrera JM, Salihagic-Kadic A, Andonotopo W. Structural and functional early human development assessed by three-dimensional and fourdimensional sonography. Fertil Steril 2005;84:1285-99.

22. Kurjak A, Andonotopo W, Hafner T, Salihagic Kadic A, Stanojevic M, Azumendi G, Ahmed B, Carrera JM, Troyano JM. Normal standards for fetal neurobehavioral developmentslongitudinal quantification by four-dimensional sonography. $\mathrm{J}$ Perinat Med. 2006;34:56-65.

23. Amiel-Tison C, Gosselin J, Kurjak A. Neurosonography in the second half of fetal life: a neonatologist's point of view. J Perinat Med 2006;34:437-46.

24. Andonotopo W, Kurjak A, Kosuta MI. Behavior of an anencephalic fetus studied by 4D sonography. J Matern Fetal Neonatal Med. 2005; 17:165-8. 